\title{
Neural and Aneural Regions Generated by the Use of Chemical Surface Coatings
}

\author{
Maria Pardo-Figuerez, ${ }^{\dagger, \ddagger}$ Neil R. W. Martin, ${ }^{\dagger}$ Darren J. Player, ${ }^{\dagger}$ Andrew J. Capel, ${ }^{\dagger, \ddagger}$
}

Steve D. R. Christie, ${ }^{\ddagger 0}$ and Mark P. Lewis, ${ }^{*}+0$

${ }^{\dagger}$ National Centre for Sport and Exercise Medicine (NCSEM), School of Sport, Exercise and Health Sciences, Loughborough

University, Epinal Way, Loughborough LE11 3TU, United Kingdom

${ }^{\ddagger}$ Chemistry Department, School of Science, Loughborough University, Epinal Way, Loughborough LE11 3TU, United Kingdom

\section{Supporting Information}

ABSTRACT: The disordered environment found in conventional neural cultures impedes various applications where cell directionality is a key process for functionality. Neurons are highly specialized cells known to be greatly dependent on interactions with their surroundings. Therefore, when chemical cues are incorporated on the surface material, a precise control over neuronal behavior can be achieved. Here, the behavior of SH-SY5Y neurons on a variety of self-assembled monolayers (SAMs) and polymer brushes both in isolation and combination to promote cellular spatial control was determined. APTES and BIBB coatings promoted the highest cell viability, proliferation, metabolic activity, and neuronal maturation, while low cell survival was seen on PKSPMA and

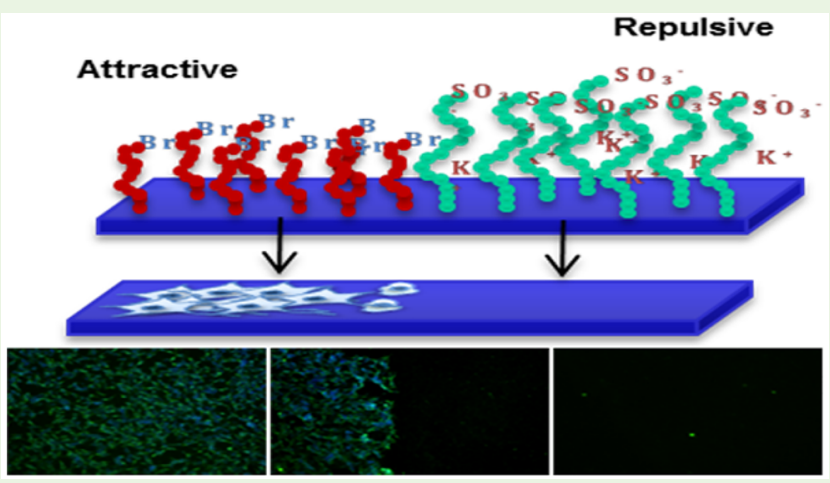
PMETAC surfaces. These cell-attractive and repulsive surfaces were combined to generate a binary BIBB-PKSPMA coating, whereby cellular growth was restricted to an exclusive neural region. The utility of these coatings when precisely combined could act as a bioactive/bioinert surface resulting in a biomimetic environment where control over neuronal growth and directionality can be achieved.

KEYWORDS: polymer brushes, SAMs, cell behavior, SH-SY5Y

\section{INTRODUCTION}

Conventional in vitro culture systems are often used to examine biological mechanisms underpinning nervous system physiology. ${ }^{1}$ In such systems, the simplicity of the homogeneous surfaces typically utilized results in a disorganized neuronal environment, where the lack of cellular directionality can affect cell functionality, ultimately limiting accuracy and reliability in in vitro neural models. ${ }^{2,3}$ A potential solution to increase neuronal functionality within in vitro systems is the use of chemically modified surfaces. This strategy allows the incorporation or modification of various chemical surface cues which will result in a change in cell response, ultimately contributing to specific cell behaviors and function.

Modifying surface parameters such as wettability, surface energy, surface charge, and chemical functionality have been known to govern cell processes such as adhesion, proliferation, directionality, and differentiation. ${ }^{4-6}$ For example, adhesion of osteoblasts was observed to decrease when surface hydrophobicity was increased. ${ }^{7}$ Likewise, neural stem cells were shown to be very sensitive to changes in the chemical functionality of the surface material. ${ }^{8}$ Polymer brushes and self-assembled monolayers (SAMs) have been used to obtain robust changes in the surface material properties, promoting a reproducible cell response., ${ }^{9,10}$ SAMs are based on tightly packed molecular assemblies attached to the surface by the adsorption of a surfactant. ${ }^{11}$ Their simple structure and easy formation make them attractive as a high throughput coating method. ${ }^{11-15}$ This technique has been used to control cell directionality ${ }^{16}$ and also as a potential tool to promote or inhibit cell adhesion, depending on the nature of the monolayer. ${ }^{17,18}$ Polymer brushes possess a more complex architecture based on long polymer chains attached by one end to a surface or interface. ${ }^{19}$ Their increased robustness and flexibility in the brush synthesis compared to SAMs allow the use of a wider range of chemical functionalities. This advantage has been utilized to obtain control over cell growth ${ }^{20}$ and as a potential method to generate antifouling surfaces. ${ }^{21,22}$ Such strategies have been successfully utilized in neuronal cell culture; however, every SAM and polymer brush is provided with a vast range of specific characteristics, and therefore, the need to test each surface is essential to avoid an unexpected cell response. $^{23}$

Received: September 7, 2017

Accepted: November 29, 2017

Published: November 29, 2017 
The SH-SY5Y human neuroblastoma cell line has been used as a robust neuronal in vitro model, ${ }^{24,25}$ demonstrating altered behaviors such as adhesion and differentiation in response to surface modifications such as plasma treatment ${ }^{26}$ or protein surface deposition. ${ }^{27}$ Although research has been conducted to assess cell behavior in various surface properties, the response between cell lineages is variable, and thus, the mechanisms behind the cell response to specific surface cues is not fully understood. $^{28}$ Therefore, there is a significant interest to show how this neuronal cell line responds to modified surfaces other than those currently used. To this end, SAMs (APTES and BIBB) and polymer brushes (PKSPMA, PMETAC, and PMMA) were used as coatings, and the effect on SH-SY5Y neuronal behavior was recorded. This set of coatings was chosen to highlight the effect of different chemical properties on neuronal behavior (functional group variation, surface charges, surface wettability, etc.) as these parameters have been associated with changes in cell behavior. ${ }^{29,30}$ The knowledge gained from this study will provide further insight into neuronsurface interactions and should demonstrate a suitable combination of bioactive and bioinert surfaces that permit precise neuronal guidance.

\section{MATERIALS AND METHODS}

2.1. Materials. The chemicals (3-aminopropyl)-triethoxysilane (APTES, >98\%), triethylamine (TEA, >99.5\%), 2-bromoisobutyryl bromide (BIBB, >98\%), copper(I) bromide ( $\mathrm{CuBr}, 99.9 \%)$, copper(II) bromide $\left(\mathrm{CuBr}_{2}, 98 \%\right), 2,2^{\prime}$-bipyridyl (Bpy, 99\%), methyl methacrylate (MMA, contains $\leq 30 \mathrm{ppm}$ MEHQ as inhibitor, 99\%), [2(methacryloyloxy)ethyl]trimethylammonium chloride solution (METAC, 80 wt \% in $\mathrm{H}_{2} \mathrm{O}$ ), (+)-sodium L-ascorbate, (crystalline, $\geq 98 \%$ ) and 3-sulfopropyl methacrylate potassium salt (KSPMA, 98\%) were purchased from Sigma-Aldrich (Welwyn Garden City, Hertforshire, UK) and used as received. Silicon wafers $(\langle 100\rangle$ orientation, boron-doped, $0-100 \Omega \cdot \mathrm{cm}, \sim 500 \mu \mathrm{m}$ thickness) were purchased from Compart Technology (Peterborough, Cambridgeshire, UK) and cut into $\sim 1 \mathrm{~cm}^{2}$ pieces for further modification. Glass slides $(1.0-1.2 \mathrm{~mm}$ thick in dimensions of $25.4 \times 76.2 \mathrm{~mm})$ were purchased from Scientific Glass Laboratories Ltd. (Canal lane, Tunstall, Stoke-on Trent, UK) and were also cut in pieces of $1-2 \mathrm{~cm}^{2}$.

For cell culture experiments, Dulbecco's modified Eagle's medium (DMEM) GlutaMAX, heat inactivated fetal bovine serum (FBS), trypan blue, and penicillin/streptomycin (PenStrep: 10000 units penicillin and $10 \mathrm{mg}$ streptomycin/mL were purchased from Gibco (Invitrogen). Trypsin, TRIzol, and the primary antibody monoclonal anti- $\beta$ tubulin III produced in mouse $(\sim 2.0 \mathrm{mg} / \mathrm{mL})$ used to assess neuronal morphology were acquired from Sigma-Aldrich. The primers to detect mRNA coding for human microtubule associated protein tau (MAPT) and human RNA Polymerase II (RPII $\beta$; Table 1) were purchased from Sigma-Aldrich. Quant-iT PicoGreen dsDNA assay, alamarBlue, and LIVE/DEAD viability/cytotoxicity assay were purchased from Invitrogen. DAPI nuclei counterstain and Alexa Fluor 488 goat antimouse IgG were obtained from Life Technologies (Thermo Scientific).

Table 1. Primer Sequences Used for RT-qPCR To Analyze the Level of Neuronal Maturation on Chemical Modified Substrates

\begin{tabular}{cccc} 
target gene & & \multicolumn{1}{c}{ sequence $5^{\prime}-3^{\prime}$} & ref. seq. number \\
MAPI & F & GAAAAATAGGCCTTGCCTTAG & NG_007398.1 \\
& R & CCTTGAGTTTCATCTCCTTTG & \\
RPII $\beta$ & F & AAGGCTTGGTTAGACAACAG & NM_000938.2 \\
& R & TATCGTGGCGGTTCTTCA &
\end{tabular}

2.2. Methods. 2.3.1. Sample Modification and Surface Characterization. The formation of hydroxyl groups on the surface, BIBB-APTES attachment, and the synthesis of PMMA, PKSPMA, and PMETAC have been previously reported. ${ }^{31-34}$ The polymerization time was $24 \mathrm{~h}$, and the brushes were prepared by surface-initiated atom transfer radical polymerization (SI-ATRP) and surface-initiated activators regenerated by electron transfer (SI-ARGET-ATRP; Figure S1).

For X-ray photoelectron spectroscopy (XPS), a Thermo Avantage software in a Thermo Scientific $\mathrm{K} \alpha$ with a monochromated $\mathrm{Al} \mathrm{K} \alpha$ Xray source $(1486.6 \mathrm{eV})$ was used. The peaks were fitted using a Shirley background. $^{35}$

Infrared spectra were measured with a PerkinElmer Spectrum 100 Fourier transform infrared (FT-IR) (Cambridge, Cambridgeshire, UK) spectrophotometer. A cleaned silicon wafer was used to determine the background signal. Spectra were taken averaging a total of 128 scans per sample run.

Ellipsometry studies were conducted using a Gaertner Scientific (Skokie, Illinois, United States) L116-B rotating analyzer ellipsometer equipped with a $1 \mathrm{~mW} 632.8 \mathrm{~nm}$ helium-neon laser at an angle of incidence of $70^{\circ}$. Three different points were measured for each sample, and the mean $( \pm S D)$ of three independent samples were calculated.

Static water contact angle was measured using $2 \mu \mathrm{L}$ drop of ultrapure water (Sigma-Aldrich) with a DataPhysics OCA 20 using a SCA 20 software. Four different samples were measured, and five values were taken from different parts of the sample. Results are expressed as mean $( \pm \mathrm{SD})$.

2.3.2. SH-SY5Y Neuronal Cell Culture. The SH-SY5Y neuroblastoma cell line (European Collection of Cell Cultures, ECACC) was subcultured in growth media (GM) consisting of DMEM GlutaMAX supplemented with $10 \% \mathrm{v} / \mathrm{v}$ heat-inactivated fetal bovine serum (FBS) and $1 \% \mathrm{v} / \mathrm{v}$ penicillin/streptomycin in an humidified $5 \%$ $\mathrm{CO}_{2}$ atmosphere at $37{ }^{\circ} \mathrm{C}$ until $80 \%$ confluent, at which point cells were detached using trypsin EDTA, counted, and plated onto chemically modified glass slides or noncoated glass slides (control). Prior to experimentation, glass slides were sterilized by incubation in $70 \%$ ethanol for at least an hour and then left to dry under sterile conditions in a biological safety cabinet.

2.3.3. Cell Viability. Cell viability was assessed using the LIVE/ DEAD assay following 1, 3, 5, and 7 days of culture. A cell density of 5000 cells $/ \mathrm{cm}^{2}$ was used, and for each time point, samples were washed twice with phosphate buffered saline (PBS) and incubated in $200 \mu \mathrm{L}$ of dye solution containing $0.5 \mu \mathrm{M}$ calcein AM and $0.5 \mu \mathrm{M}$ ethidium homodimer ${ }^{-1}$ in PBS. The samples were then incubated in the dark at room temperature for $45 \mathrm{~min}$. Thereafter, the solution was pipetted away, and the samples were mounted onto a glass coverslips using Fluoromount Aqueous Mounting Medium. Five random fields of each sample were imaged on both green and red channels, and the number of live and dead cells was counted with Image J (1.50a) cell counter plugin. The number of live cells divided by the total number of live and dead cells was defined as the fractional viability.

2.3.4. Assessment of Metabolic Activity. alamarBlue was used to study the metabolic activity of SH-SY5Y on chemically modified surfaces during $1,3,5$, and 7 days in cell culture. SH-SY5Y were seeded at $10000 \mathrm{cells} / \mathrm{cm}^{2}$, and for every time point, the medium from the samples was aspirated off and the substrates were washed with PBS twice. A solution of $10 \% \mathrm{v} / \mathrm{v}$ alamarBlue in serum free DMEM GlutaMAX was added into each well and incubated for $4 \mathrm{~h}$ at $37^{\circ} \mathrm{C}$. Thereafter, $100 \mu \mathrm{L}$ of the supernatant was pipetted into a black opaque 96-well plate, and fluorescence was measured in a Thermo scientific Varioskan Flash plate reader with excitation/emission set at $560 / 590 \mathrm{~nm}$. The fluorescence intensity was correlated with the cell metabolic activity. ${ }^{36,37}$

2.3.5. Assessment of Proliferation. Quant-iT PicoGreen dsDNA assay was used to assess proliferation at day $1,3,5$, and 7 . A cell density of 10000 cells $/ \mathrm{cm}^{2}$ was seeded and lysed with $0.1 \%$ Triton X100 in pure water and collected in nuclease-free vials after each time point. Vials were vortexed, and $100 \mu \mathrm{L}$ of lysate was pipetted into black flat-bottomed 96-well plates along with $100 \mu \mathrm{L}$ of Quant-iT 


\begin{tabular}{|c|c|c|c|c|}
\hline \multirow[t]{2}{*}{ Modification step } & & \multicolumn{3}{|c|}{ Atomic composition (\%) } \\
\hline & Si2p & \multicolumn{2}{|c|}{ C1s } & 01s \\
\hline Clean glass slide (SiOH) & 28.13 & \multicolumn{2}{|c|}{4.59} & 56.39 \\
\hline Aminosylation ( $\left.\mathrm{Si}-\mathrm{NH}_{2}\right)$ & 26.39 & \multicolumn{2}{|c|}{20.02} & 47.17 \\
\hline Initiator (Si-Br) & 27.96 & \multicolumn{2}{|c|}{10.95} & 54.17 \\
\hline \multirow{5}{*}{ B } & Polymer brush & \multicolumn{3}{|c|}{ Atomic composition (\%) } \\
\hline & & C1s & $01 \mathrm{~s}$ & Si2p \\
\hline & PMMA & 26.02 & 72.33 & 1.65 \\
\hline & PKSPMA & 27.2 & 61.76 & 0.58 \\
\hline & PMETAC & 18.98 & 65.67 & 4.05 \\
\hline
\end{tabular}

Figure 1. (A) Atom composition (\%) from XPS measurements on the cleaning, aminosylation, and initiator steps. Only the main atoms are presented for clarity. (B) Atom composition (\%) of C 1s, O 1s, and Si 2p from XPS measurements on the polymer brushes.

PicoGreen working solution (1:200 from $1 \times$ TE buffer). Plates were incubated in the dark for $5 \mathrm{~min}$ at room temperature, and the fluorescence given by PicoGreen was measured in a Thermo Scientific Varioskan Flash microplate reader at excitation and emission wavelengths of 480 and $520 \mathrm{~nm}$, respectively. Indirect quantification of DNA was performed by a dsDNA standard curve.

2.3.6. Neurite Outgrowth and MAPT Gene Expression. For differentiation experiments, 5000 cells $/ \mathrm{cm}^{2}$ were seeded onto the surface modified samples and cultured on GM until a 50\% confluence was reached. Thereafter, cells were treated with differentiation media (DM) consisting of GM with $10 \mu \mathrm{M}$ of all-trans retinoic acid (RA). Neurite length was measured with image J by tracing a segmented line across the neurite (Figure 5A) before DM was added (PRE RA time point) and then after $24 \mathrm{~h}$ in DM. For gene expression experiments, samples were cultured in GM until 50\% confluence was reached and subsequently treated with DM for 3 days.

2.3.7. RNA Extraction and RT-qPCR. RNA was extracted using TRIzol pursuant to the manufacturer's instructions (Sigma-Aldrich) and quantified spectrophotometrically using a NanoDrop 2000 (Thermo Scientific, UK). The amplification of RT-qPCR was assessed by using QuantiFast SYBR Green one step kit (Qiagen) on a ViiA7 Real-Time PCR System (Applied Biosystems, Life Technologies) using associated software. Reactions were prepared in 384-well plates and consisted of $20 \mathrm{ng}$ of RNA in $5 \mu \mathrm{L}$ and $5 \mu \mathrm{L}$ of Master mix consisting of $4.7 \mu \mathrm{L}$ of SYBR Green, $0.1 \mu \mathrm{L}$ of both forward and reverse primer, and $0.1 \mu \mathrm{L}$ of quantifast reverse transcriptase kit. The reaction was carried out as follows: $50{ }^{\circ} \mathrm{C}$ for $10 \mathrm{~min}, 95^{\circ} \mathrm{C}$ for $5 \mathrm{~min}$, followed by 40 cycles of $95{ }^{\circ} \mathrm{C}$ for $10 \mathrm{~s}$ and $60{ }^{\circ} \mathrm{C}$ for $30 \mathrm{~s}$. Data were analyzed using the $2^{-\Delta \Delta C}{ }_{T}$ method ${ }^{38}$ and normalized to $\mathrm{RPII} \beta$ and expressed relative to a single APTES sample.

2.3.8. Immunostaining. Cells were fixed with $3.7 \%$ paraformaldehyde diluted in PBS and subsequently blocked and permeabilized with $5 \%$ goat serum (GS) and $0.2 \%$ Triton X-100 in $1 \times$ Tris buffered saline (TBS) for $30 \mathrm{~min}$ at room temperature. Thereafter, a solution with monoclonal mouse anti- $\beta$-tubulin III $(1 / 200 \mathrm{v} / \mathrm{v})$ antibody and $2 \% \mathrm{GS}$ in TBS was added and incubated for $2 \mathrm{~h}$. This solution was aspirated off, and AlexaFluor488-conjugated goat anti-mouse IgG (1/200 v/v) was added with nuclear counter stain DAPI $(1 / 1000 \mathrm{v} / \mathrm{v})$ containing $2 \%$ GS and $0.2 \%$ Triton X-100 in TBS for $1 \mathrm{~h}$. After being thoroughly rinsed with PBS, cell culture samples were mounted onto cover slides using Fluoromount Aqueous Mounting Medium and imaged using a LEICA DM2500 fluorescence microscope.

2.3.9. Statistical Analysis. All of the data are presented as the mean $( \pm \mathrm{SD})$. Repeated measure two-way ANOVA was used to analyze the proliferation, metabolic activity, and differentiation data determined by Quant-iT PicoGreen, alamarBlue, and average neurite length data, respectively. Bonferroni's posthoc test was used for multiple comparison tests to determine differences among the different surfaces over time. One-way ANOVA was performed for the cell viability results followed by a Bonferroni's posthoc test. GraphPad commercial software 6.0 was utilized to run statistics. Differences in MAPT gene expression between control, APTES, and BIBB were analyzed using one-way ANOVA. For MAPT gene expression of BIBB and APTES, an unpaired $t$ test was used to detect differences between conditions. Differences were considered statistically significant when $p \leq 0.05$.

\section{RESULTS}

3.1. Surface Preparation and Characterization. Surface modification began with the oxidation of the glass surface via UV/ozone treatment, which removed the majority of the organic contaminants as well as promoting the formation of a uniform hydroxyl $(-\mathrm{OH})$ layer on the surface (indicated as $\mathrm{SiOH}$, see Figure 1A). Thereafter, APTES attachment was carried out by the formation of $\mathrm{Si}-\mathrm{O}-\mathrm{Si}$ bonds on the surface, followed by the anchoring of BIBB initiator, which would in turn allow the synthesis of the polymer brushes (PMMA, PKSPMA, and PMETAC) by surface initiated polymerization (SIP). After each step, XPS was used to analyze the chemical surface composition of each coating.

XPS analysis of the $\mathrm{SiOH}$ layer showed the silicon ( $\mathrm{Si} 2 \mathrm{p}$ ) and oxygen (O 1s) peaks as the most predominant elements on the surface. This was anticipated as these two atoms are the characteristic elements of glass surfaces (Figure 1A, $\mathrm{SiOH}$ clean glass slide). APTES attachment showed an increase in the carbon percentage (C 1s) along with the presence of a new nitrogen peak $\sim 400 \mathrm{eV}$ with a $1.6 \%$ of nitrogen content $(\mathrm{N} \mathrm{1s})$ (Figure 1A, aminosylation), indicating the successful addition of amine and propyl groups onto the surface. In addition, a more hydrophobic behavior was found after APTES anchoring, indicating a clear change in the surface chemistry of the substrate (Figure S2). The introduction of BIBB onto the APTES surface was confirmed by the presence of a bromide group $(\mathrm{Br} 3 \mathrm{~d}$ ), along with an increase in $\mathrm{C} 1 \mathrm{~s}$ content (Figure $1 \mathrm{~A}$, initiator). Furthermore, a more hydrophobic surface was obtained after BIBB deposition (Figure S2). It is noteworthy to comment that there was an increase in oxygen content ( $\mathrm{O} 1 \mathrm{~s})$ compared to APTES, probably due to the introduction of $\mathrm{C}=$ O groups in this structure.

After the covalent attachment of a BIBB initiator, the grafting of the brushes was carried out to obtain PMETAC, PKSPMA, and PMMA layers. XPS analysis of the brushes indicated a drastic reduction of the $\mathrm{Si} 2 \mathrm{p}$ signal when compared with the percentage of $\mathrm{Si} 2 \mathrm{p}$ showed in the clean glass slides $(\mathrm{SiOH})$, suggesting that the glass surface was being covered by a new 
polymer layer, which resulted in the attenuation of the main surface signal (Figure 1B). This was further confirmed when thick polymer layers with heights ranging between the 60-100 $\mathrm{nm}$ were obtained by ellipsometry (Figure S3). The formation of the polymer brushes also showed different wettabilities, with PMETAC and PKSPMA surfaces rendering a very hydrophilic behavior, whereas PMMA brushes gave a slight increase in water contact angle toward hydrophobicity (Figure S2).

Further surface chemical analysis of the polymer was also analyzed by FT-IR (Figure S4). The spectra showed various peaks found around $3000-2800 \mathrm{~cm}^{-1}$ for PKSPMA, PMMA, and PMETAC attributed to the stretching of $\mathrm{C}-\mathrm{H}$ groups. Furthermore, the characteristic $\mathrm{C}=\mathrm{O}$ stretching peak of acrylate polymers appeared at $\sim 1780-1720 \mathrm{~cm}^{-1}$ for all the brushes, suggesting that the polymerization was successful (see Figure S4). In PKSPMA samples, strong peaks were found at a range of $\sim 1050$ and $\sim 1190 \mathrm{~cm}^{-1}$. These corresponded to the asymmetric and symmetric stretching of the sulfonate group, respectively. In PMMA, the peak $\sim 1150 \mathrm{~cm}^{-1}$ was assigned to the $\mathrm{C}-\mathrm{O}$ of the ester. In PMETAC, peaks arising at $\sim 1200$ $\mathrm{cm}^{-1}$ were assigned to the $\mathrm{C}-\mathrm{O}$ ester groups. The different peaks found in these spectra indicate the main functional groups of the brushes, suggesting the successful formation of the brushes. The black spectrum corresponded to a noncoated silicon wafer, which main peak corresponds to the $\mathrm{Si}-\mathrm{O}$ asymmetric stretching mode of the silicon. Overall, all these analytical techniques together supported the presence of these coatings on the surface.

3.2. Effect of Chemical Surface Modifications on Cellular Viability and Proliferation of SH-SY5Y Neurons. Cellular viability was tested on chemically modified glass at days 1, 3, 5, and 7 (Figures 2 and S5). While cell viability was high on the majority of chemical surfaces through all the culture period, neurons cultured on PMETAC showed a reduced viability through all time points investigated, suggesting that the coating may have a degree of toxicity, an effect which became more pronounced over time $(p<0.05$ after 3 days in culture, Figure 3). Furthermore, although cell viability was similar for all the coatings with the exception of PMETAC, the amount of cells adhered to these substrates appeared different (see Figure S6 for cell adhesion data on these surfaces). This was the case especially for the PKSPMA surface, where viability seemed as high as in the other coatings; however, SH-SY5Y cells did not appear to have a preference for this surface, avoiding adhesion. Additionally, PKSPMA showed a slightly reduction in viability at day 7; however, this was mainly due to the poor cell attachment on the surface, which made the quantification of cell viability for this coating very dependent on the amount of cells presented on the surface (Figure S6).

Metabolic activity and cellular proliferation were quantified on these surfaces via alamarBlue (Figure S7) and Quant-iT PicoGreen assays (Figure 4), respectively. Using alamarBlue, the fluorescence intensity showed a nonsignificant trend where fluorescence was low in PKSPMA and PMETAC coatings when compared to the other coatings after $24 \mathrm{~h}$. This was followed by a noticeable decrease in fluorescence through the entire cell culture period, indicating that the metabolic activity of the cells on these coatings was reduced ( $p>0.05$ for all the time points but day 7 when compared to noncoated glass). Metabolic activity continued to rise on BIBB and noncoated glass during the time course ( 3,5 , and 7 days), while a plateau in fluorescence intensity during 3-5 days occurred for the APTES coatings, followed by an increase in fluorescence toward the
DAY 1
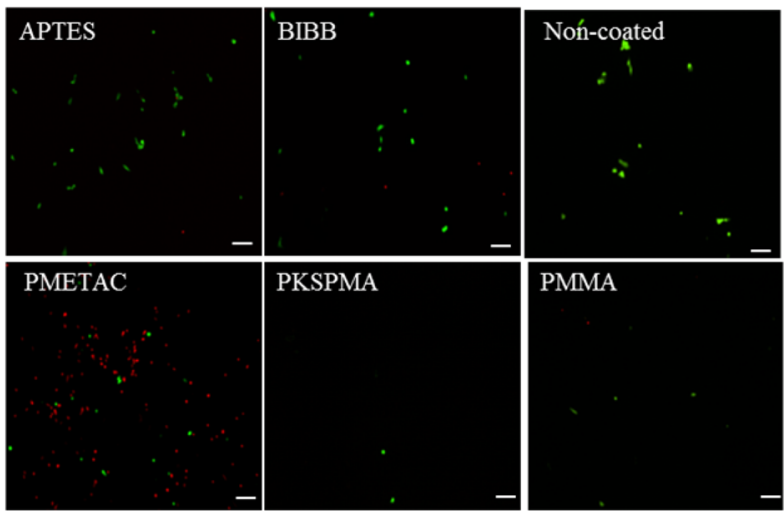

DAY 7
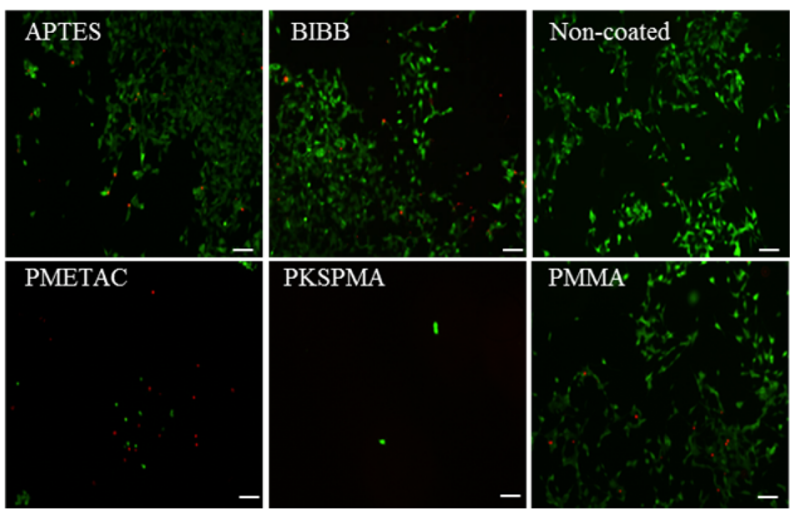

Figure 2. Representative fluorescence microscopy images of live/dead staining on SH-SY5Y after 1 and 7 days on chemical surfaces. Cell viability images at day 3 and 5 can be found in the Supporting Information, Figure S5. The staining is represented by calcein AM for live cells (green) and ethidium homodimer ${ }^{-1}$ for dead cell nuclei (red). Noncoated indicates untreated glass. Scale bar $=100 \mu \mathrm{m}$.

end of day 7 (see Figure S7, $p>0.05$ ). The metabolic activity of cells seeded on PMMA samples did not seem to increase significantly during the course of the experiment. This tendency was also noticed when examining phase contrast micrographs (Figure S8), where a similar area covered by cells on noncoated glass, APTES, and BIBB was observed, while detached cells and clumps could be observed in cells cultured on PKSPMA and PMETAC surfaces. Overall, a trend with a reduced metabolic activity was found for PKSPMA, PMETAC, and PMMA, whereas APTES and BIBB showed a metabolic activity similar to the one found on noncoated glass. Similarly, data presented from Quant-iT PicoGreen assay showed that APTES, BIBB, and noncoated glass exhibited the highest proliferation rates over the 7 day culture period, increasing from day 1 until the end of the experiment (Figure 4). There seemed to be an increase in proliferation on cells cultured on PMETAC and PKSPMA; however, the rates remained low compared to those on noncoated glass $(p<0.05$ from day 3 to 7$)$. The degree of proliferation on PMMA samples increased exponentially until day 5 and then remained stable until day 7 (Figure 4).

These results indicate that APTES and BIBB are comparable to control glass in terms of cellular proliferation and metabolic activity, while substrates such as PKSPMA and PMETAC were not able to obtain viable cells to adhere and proliferate.

3.3. Effect of Surface Coatings on Neurite Outgrowth and Differentiation of SH-SY5Y Cells. Neurite outgrowth was measured in cells cultured on both SAMs and polymer 

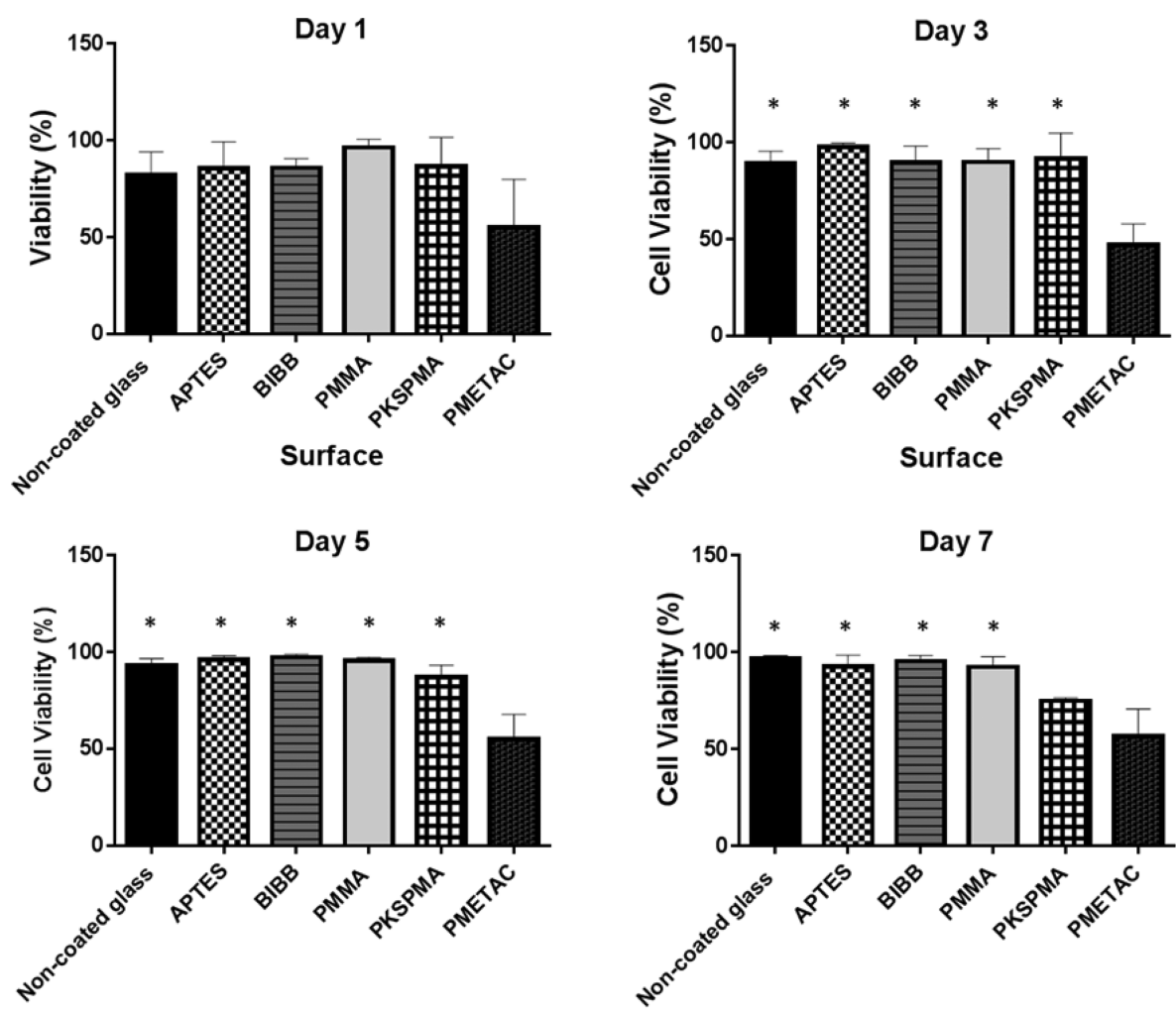

Surface

Surface

Figure 3. SH-SY5Y cell viability (\%) on the different chemical coatings (APTES, BIBB, PMMA, PKSPMA, PMETAC, and noncoated glass) at days $1,3,5$, and 7. Error bars represent the SD of the mean. High cell viability was presented in all the coatings throughout the time course when compared to noncoated glass, except for PMETAC. Statistical analyses were assessed by one-way ANOVA within groups, followed by a Bonferroni posthoc test. Asterisks indicate statistical differences between the substrates vs PMETAC $(p<0.05, n=3)$.

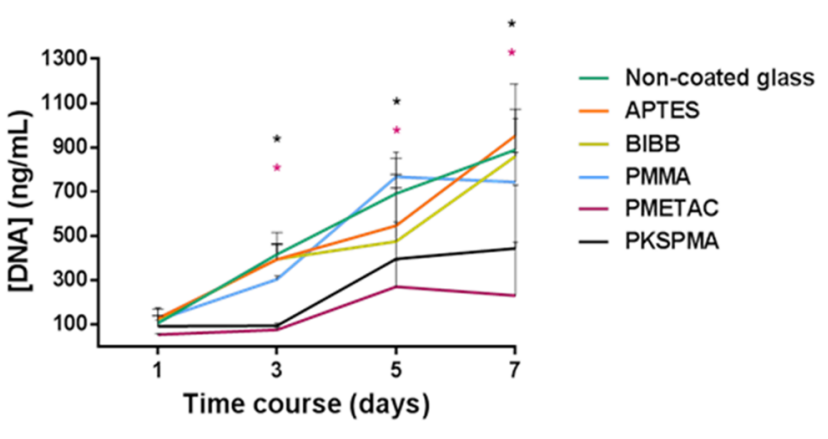

Figure 4. Proliferation rates assessed by DNA quantification from Quant-iT PicoGreen fluorescence of SH-SY5Y cells. Cells were lysed at days 1, 3, 5, and 7 for the different surfaces. Error bars represent the $\mathrm{SD}$ of the mean. Asterisks (which have been colored in accordance with the color legend) indicate statistical differences between the substrates vs noncoated glass $(* p<0.05, n=3)$.

brushes to study whether the presence of these coatings could trigger intracellular signals leading to neurite extension. Thus, neurite length was measured on the different surfaces before the differentiating agent all-trans retinoic acid was added (PRE $\mathrm{RA}$ ) and then after $24 \mathrm{~h}$ in DM containing RA. Results indicate that neurons cultured on APTES and BIBB surfaces had, on average, neurites of significantly greater length than those cultured on noncoated glass before the differentiating agent was added. This suggests a major level of neuronal differentiation on these modified substrates, even prior to the addition of RA (Figure 5A, $p<0.05$ ). After $24 \mathrm{~h}$ of adding retinoic acid (DM), an overall increase in neurite length on all modified surfaces was observed with a greater neurite outgrowth in neurons cultured on APTES and BIBB (Figure 5A, $p<0.05$ for BIBB and $p=0.06$ for APTES). Measures on PKSPMA and PMETAC samples could not be assessed due to the lack of cell survival, adhesion, and therefore differentiation, supporting previous observations. PMMA remained similar to noncoated glass with no significant differences before and after addition of RA $(p>0.05)$. To identify the single, optimum coating for neuronal survival and maturation, the expression of MAPT by cells cultured on noncoated glass (CTRL), APTES, and BIBB was determined. There was an increase in MAPT expression for noncoated glass compared to APTES and BIBB, although the differences did not reach significance. MAPT mRNA expression was higher in neurons cultured on BIBB compared to APTES (Figure $5 \mathrm{~B}, p=0.06$ ), which may indicate a greater presence of neuronal maturation in neurites presented on BIBB vs APTES, although with nonsignificant differences.

3.4. Effect of Binary Coating on SH-SY5Y Cells. The differentiation, viability, and proliferation data commented on above clearly demonstrated that APTES and BIBB coatings promoted cellular growth and differentiation, while PKSPMA appeared to inhibit these parameters without inducing any toxic effect. As such, it was investigated whether fabricating a binarycoated surface (half BIBB/half PKSPMA) would lead to tightly controlled growth without any visible negative effects. A binarycoated sample was achieved by submerging half of a BIBB initiator sample (necessary for the formation of the brush) into the PKSPMA monomer solution under nitrogen overnight. XPS analysis showed a difference in the percentage composition of Si $2 p$ between BIBB and PKSPMA, suggesting 
A
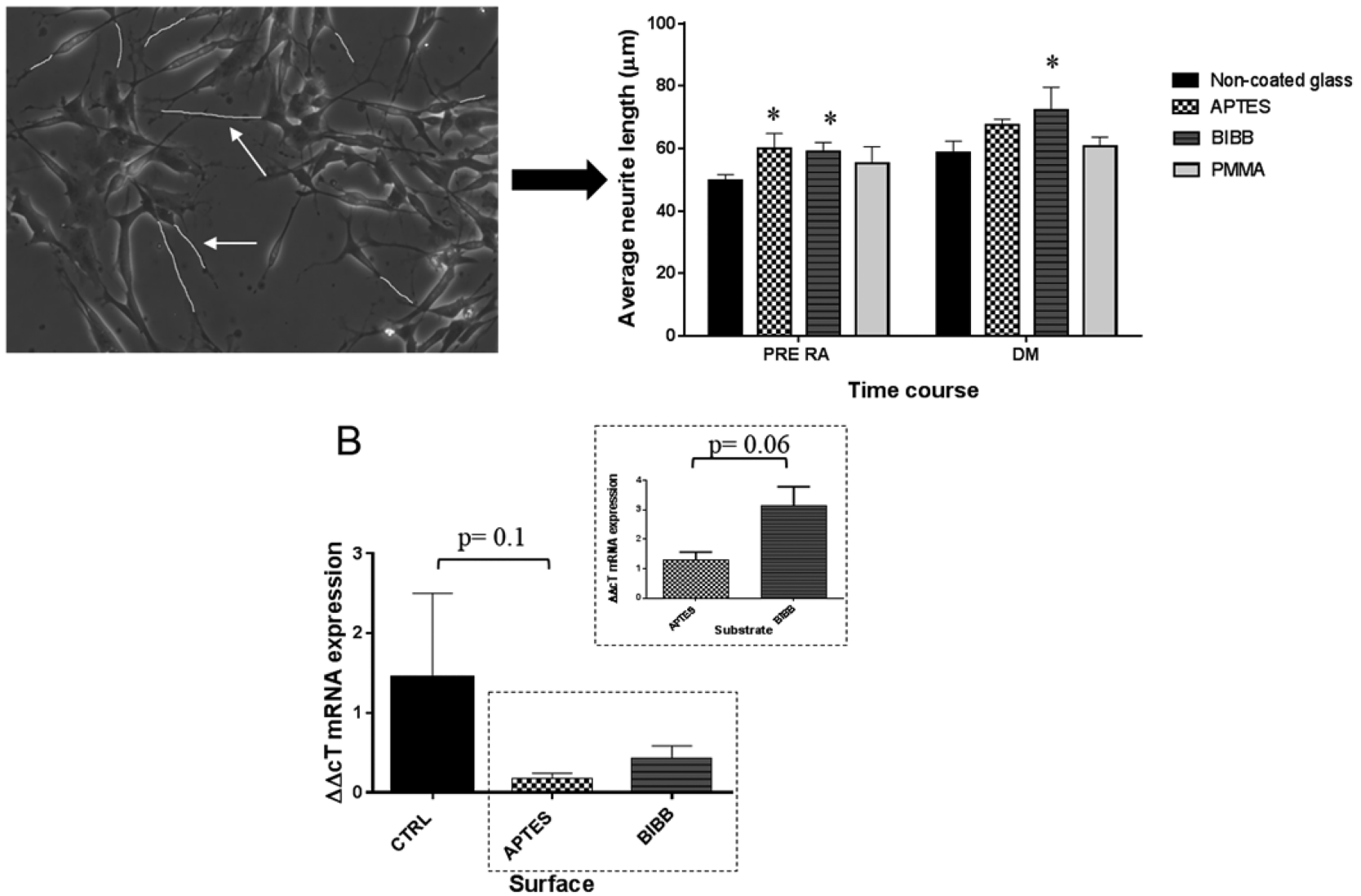

Figure 5. (A) Representation of neurite measurement by tracing a line across the neurite with Image $\mathrm{J}$ software and average neurite length found on APTES, BIBB, PMMA and noncoated glass before and after all-trans RA was added. An increase in average neurite length was observed in APTES and BIBB coatings when compared to that on noncoated glass before (PRE RA) and after $24 \mathrm{~h}$ of adding RA for BIBB (DM). Error bars correspond to SD of the mean. Asterisks represent significant differences when compared with noncoated glass $(* p<0.05, n=3)$. (B) Graph illustrates the expression of MAPT gene (codes for tau protein involved in microtubule stabilization) on SH-SH5Y cells cultured on noncoated glass (CTRL), APTES, and BIBB substrates $(n=3)$. Error bars correspond to the SD of the mean.

that PKSPMA had been successfully coated on that side of the sample because the Si $2 p$ signal from the glass surface was more attenuated compared to that of BIBB, indicating the presence of a thicker layer on the PKSPMA area (Figure S9). The PKSPMA zone had $27 \%$ more $\mathrm{C}$ 1s content than BIBB, which was comprehensible due to the presence of more $\mathrm{C}-\mathrm{C}, \mathrm{C}-\mathrm{O}$, and $\mathrm{C}=\mathrm{O}$ bonds in the polymer brush. In addition, the characteristic peak of $S 2 p$ presented on PKSPMA brushes was found only on the side where the brush had been intentionally grown (Figure S9, XPS graph). Furthermore, the peak of Br 3d was present only in the BIBB zone (Figure S9, XPS graph), indicating that the initiator was covering that zone of the sample. These results together indicated that PKSPMA was present on the desired part of the sample, while the remaining part contained the functionalized BIBB initiator. Furthermore, the change on the surface chemistry was also demonstrated by the different wettability presented on both sides of the samples (Figure 6), representing the hydrophobic BIBB initiator and the hydrophilic PKSPMA brush.

SH-SY5Y cells were then evenly seeded across the binary coating. After a week in GM, the cells in the BIBB zone were confluent, whereas no cells were observed in the PKSPMA region, resulting in clear cellular partitioning and restricted cell growth (Figure 7). The presence of a PKSPMA region did not appear to negatively affect the cells in the BIBB region, further supporting the previous results indicating that PSKPMA restricts cell growth without any toxic side effects.

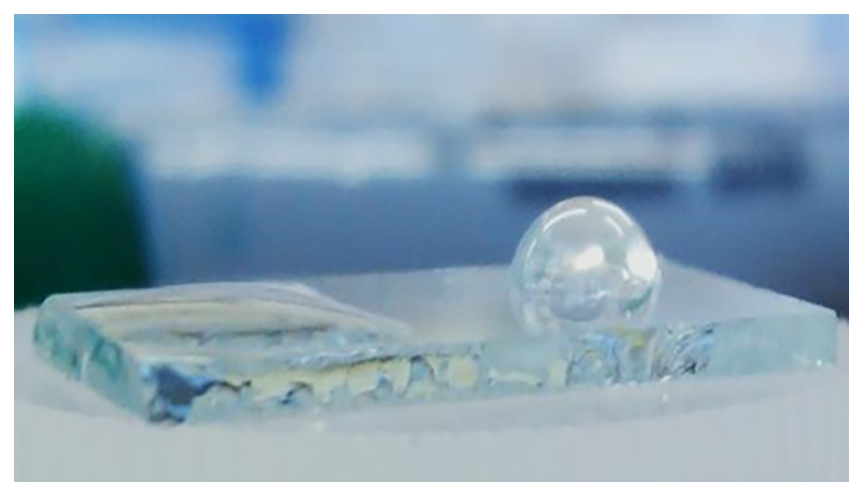

Figure 6. A macroscopic image showing the different hydrophilicity after PKSPMA polymerization, indicating the presence of both coatings (left, hydrophilic part PKSPMA; right, hydrophobic part $\mathrm{BIBB})$.

\section{DISCUSSION}

Parameters involved in cell-substrate interactions such as film architecture, ${ }^{4}$ surface charge, ${ }^{9}$ wettability, ${ }^{29}$ chemical functionality, ${ }^{18}$ and protein adsorption ${ }^{15}$ have been known to alter cell response. Therefore, there is a wide range of factors involved in triggering cell response when using chemically modified surfaces.

In neuronal cultures, surface wettability has been related to optimum neural cell adhesion with a greater neurite formation 


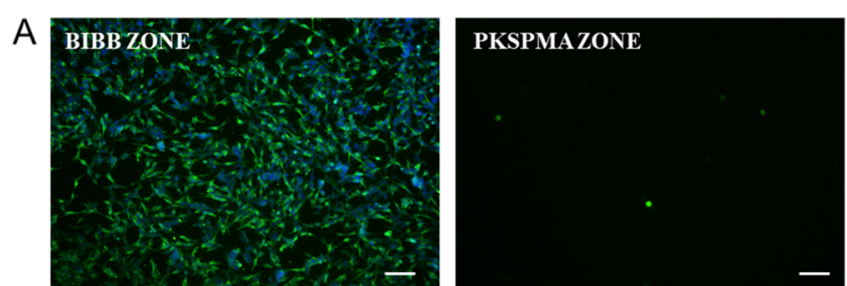

B

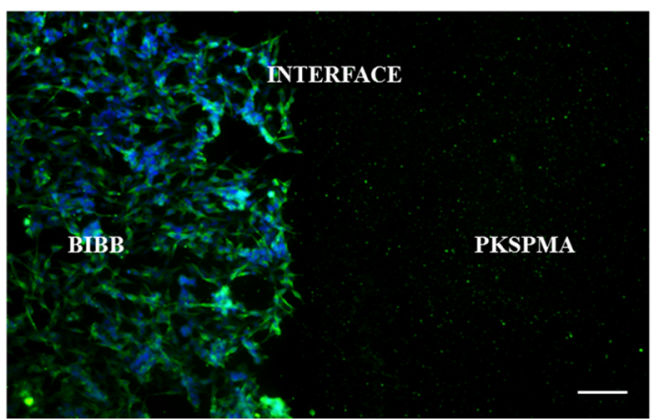

Figure 7. SH-SY5Y cells cultured on the BIBB/PKSPMA binary coating for a week. (A) Representative images of the BIBB and PKSPMA zones, illustrating the different areas of the sample. (B) Representative image of the interface between both coatings where SH-SY5Y cells remained in the BIBB area, avoiding migration toward the PKSPMA zone. Fixed cells were labeled with $\beta$-tubulin III (green), and nuclei were labeled with counterstain DAPI (blue). Scale bar = $100 \mu \mathrm{m}$.

on surfaces with a moderate hydrophilicity. ${ }^{39}$ The requirement of moderate hydrophilicity to obtain a positive neuronal response could explain why very hydrophilic surfaces such as PMETAC and PKSPMA did not succeed in obtaining neuronal adhesion, whereas APTES was shown to promote cell adhesion and proliferation. This parameter was also associated with the greater formation of neurites with increasing hydrophilicity, ${ }^{39}$ which is in agreement with the greater neurite extension found on APTES samples. However, this trend cannot be connected with cell behavior on BIBB samples, as this surface is hydrophobic. In addition, the different wettability is also related to the ability of the surface to adsorb proteins from the serum, ${ }^{7,29}$ in turn facilitating cell adhesion. This parameter is therefore another factor to consider when assessing why different substrates inhibit or favor neuronal behavior. ${ }^{39}$ Electrostatic interactions, not only between these adsorbed proteins and the coatings but also between the coatings and the cell membrane, will likely contribute as well to the different cell behavior. $^{40,41}$ This could perhaps explain why the charged surfaces of PKSPMA and PMETAC inhibited cell adhesion. Particularly for PMETAC, the positively charged substrates presented on this brush have been known to induce toxicity by interaction with charges in the cell membrane. ${ }^{42,43}$

Although it is not clear what the exact mechanisms that control the neuron-chemical-substrate interactions are, ${ }^{8,44}$ the attractive and inhibitory responses that these can achieve are of great importance for the development of novel biodevices by the manipulation of both bioactive and bioinert surfaces. In this work, both responses were accomplished in a single system, attaining control of neuronal growth in a desired area of the system and therefore showing clear cellular affects. With the appropriate engineering techniques, both substrates could be incorporated into a more complex system whereby neurons can be positioned in specific areas to study cell-cell connections for underpinning neuronal interfaces.

\section{CONCLUSIONS}

Use of polymer brushes and self-assembled monolayers in cell culture applications displays a wide range of advantages, as their properties can be tuned to promote or even inhibit cellular differentiation, proliferation, and survival. ${ }^{45}$ In this current work, SH-SY5Y cells were cultured on a wide range of polymer coatings, resulting in contrasting cellular responses. Cells cultured on APTES and BIBB displayed a greater neurite outgrowth, cell proliferation, and survival, whereas there was a lack of cell adhesion and impaired growth on PKSPMA and PMETAC brushes. Nonapparent toxic effects were shown on PKSPMA; however, a certain degree of toxicity was observed on PMETAC. When both BIBB and PKSPMA were coated on a single slide, cells adhered and grew only on the BIBB half of the slide with no apparent toxic effects of the PKSPMA. The permissive and nonpermissive cell coatings identified in these results could be incorporated into a more complex structure by using appropriate engineering techniques, resulting in a more accurate study of different cell interactions for systems such as neurons and astrocytes in the brain and also neurons and their interaction with innervating targets. Overall, this report indicates that neuronal behavior in vitro is at least partly dependent on the interaction between the cell and the chemical features of the surface. Furthermore, there is a wide range of applications for these promising coatings to be included in the biomaterial world, presenting significant progress in the control of the cell-material crosstalk.

\section{ASSOCIATED CONTENT}

\section{Supporting Information}

The Supporting Information is available free of charge on the ACS Publications website at DOI: 10.1021/acsbiomaterials.7b00663.

Chemical structures of the brushes (Figure S1), water contact angle data (Figure S2), ellipsometry measures of the brushes (Figure S3), characterization by FT-IR (Figure S4), live/dead staining at days 3 and 5 (Figure S5), data on cell adherence for each surface (Figure S6), metabolic activity data of the SH-SY5Y for each surface coating (Figure S7), contrast microscope images at different time points to assess proliferation (Figure S8), and XPS data regarding the binary BIBB/PKSPMA sample (Figure S9) (PDF)

\section{AUTHOR INFORMATION}

\section{Corresponding Author}

*E-mail: M.P.Lewis@lboro.ac.uk.

\section{ORCID}

Maria Pardo-Figuerez: 0000-0003-4856-8220

Neil R. W. Martin: 0000-0002-1988-047X

Andrew J. Capel: 0000-0002-3043-0170

Steve D. R. Christie: 0000-0002-6756-499X

Mark P. Lewis: 0000-0002-8430-4479

Notes

The authors declare no competing financial interest.

\section{ACKNOWLEDGMENTS}

We thank Loughborough University and EPSRC (Grant EP/ L02067X/1) for financially supporting this project. 


\section{REFERENCES}

(1) Gordon, J.; Amini, S.; White, M. K. General Overview of Neuronal Cell Culture. Methods Mol. Biol. 2013, 1078, 1-8.

(2) Chen, Z.; Kang, L.; Wang, Z.; Xu, F.; Gu, G.; Cui, F.; Guo, Z. Recent progress in the research of biomaterials regulating cell behavior. RSC Adv. 2014, 4 (109), 63807-63816.

(3) Charest, J. L.; Eliason, M. T.; Garcia, A. J.; King, W. P. Combined microscale mechanical topography and chemical patterns on polymer cell culture substrates. Biomaterials 2006, 27 (11), 2487-2494.

(4) Ren, T.; Mao, Z.; Guo, J.; Gao, C. Directional migration of vascular smooth muscle cells guided by a molecule weight gradient of poly(2-hydroxyethyl methacrylate) brushes. Langmuir 2013, 29, 6386-6395.

(5) Raynor, J. E.; Capadona, J. R.; Collard, D. M.; Petrie, T. A.; García, A. J. Polymer brushes and self-assembled monolayers: Versatile platforms to control cell adhesion to biomaterials (Review). Biointerphases 2009, 4 (2), FA3-A16.

(6) Kobayashi, M.; Terayama, Y.; Yamaguchi, H.; Terada, M.j Murakami, D.; Ishihara, K.; Takahara, A. Wettability and antifouling behavior on the surfaces of superhydrophilic polymer brushes. Langmuir 2012, 28, 7212-7222.

(7) Wei, J.; Igarashi, T.; Okumori, N.; Igarashi, T.; Maetani, T.; Liu, B.; Yoshinari, M. Influence of surface wettability on competitive protein adsorption and initial attachment of osteoblasts. Biomed. Mater. 2009, 4, 45002.

(8) Ren, Y. J.; Zhang, H.; Huang, H.; Wang, X. M.; Zhou, Z. Y.; Cui, F. Z.; An, Y. H. In vitro behavior of neural stem cells in response to different chemical functional groups. Biomaterials 2009, 30 (6), 10361044.

(9) Palyvoda, O.; Bordenyuk, A. N.; Yatawara, A. K.; McCullen, E.; Chen, C. C.; Benderskii, A. V.; Auner, G. W. AC Molecular organization in SAMs used for neuronal growth. Langmuir 2008, 24, 4097-4106.

(10) Moroni, L.; Gunnewiek, M. K.; Benetti, E. Polymer brush coatings regulating cell behavior: Passive interfaces turn into active. Acta Biomater. 2014, 10, 2367-2378.

(11) Ulman, A. Formation and Structure of Self-Assembled Monolayers. Chem. Rev. 1996, 96 (4), 1533-1554.

(12) Cooper, E.; Wiggs, R.; Hutt, D. A.; Parker, L.; Leggett, G. J.; Parker, T. L. Rates of attachment of fibroblasts to self-assembled monolayers formed by the adsorption of alkylthiols onto gold surfaces. J. Mater. Chem. 1997, 7, 435-441.

(13) Sukenik, C. N.; Balachander, N.; Culp, L. A.; Lewandowska, K.; Merritt, K. Modulation of cell adhesion by modification of titanium surfaces with covalently attached self-assembled monolayers. J. Biomed. Mater. Res. 1990, 24, 1307-1323.

(14) Roach, P.; Parker, T.; Gadegaard, N.; Alexander, M. R. Surface strategies for control of neuronal cell adhesion: A review. Surf. Sci. Rep. 2010, 65, 145-173.

(15) Senaratne, W.; Andruzzi, L.; Ober, C. K. Self-assembled monolayers and polymer brushes in biotechnology: Current applications and future perspectives. Biomacromolecules 2005, 6, 2427-2448

(16) Lopez, G.; Albers, M. Convenient methods for patterning the adhesion of mammalian cells to surfaces using self-assembled monolayers of alkanethiolates on gold. J. Am. Chem. Soc. 1993, 115 (12), 5877-5878.

(17) Jans, K.; Van Meerbergen, B.; Reekmans, G.; Bonroy, K.; Annaert, W.; Maes, G.; Engelborghs, Y.; Borghs, G.; Bartic, C. Chemical and biological characterization of thiol SAMs for neuronal cell attachment. Langmuir 2009, 25 (8), 4564-4570.

(18) Faucheux, N.; Schweiss, R.; Lützow, K.; Werner, C.; Groth, T. Self-assembled monolayers with different terminating groups as model substrates for cell adhesion studies. Biomaterials 2004, 25 (14), 27212730.

(19) Milner, S. T. Polymer brushes. Science 1991, 251, 905-914.

(20) Zhou, Z.; Yu, P.; Geller, H. M.; Ober, C. K. Biomimetic polymer brushes containing tethered acetylcholine analogs for protein and hippocampal neuronal cell patterning. Biomacromolecules 2013, 14, 529-537.

(21) Kuzmyn, A. R.; de los Santos Pereira, A.; Pop-Georgievski, O.; Bruns, M.; Brynda, E.; Rodriguez-Emmenegger, C. Exploiting end group functionalization for the design of antifouling bioactive brushes. Polym. Chem. 2014, 5 (13), 4124-4131.

(22) Zhao, C.; Li, L.; Wang, Q.; Yu, Q.; Zheng, J. Effect of film thickness on the antifouling performance of poly(hydroxy-functional methacrylates) grafted surfaces. Langmuir 2011, 27, 4906-4913.

(23) Tong, Y. W.; Shoichet, M. S. Enhancing the neuronal interaction on fluoropolymer surfaces with mixed peptides or spacer group linkers. Biomaterials 2001, 22 (10), 1029-1034.

(24) Xie, H.; Hu, L.; Lim, G. SH-SY5Y human neuroblastoma cell line: in vitro cell model of dopaminergic neurons in Parkinson's disease. Chin. Med. J. (Engl). 2010, 123 (8), 1086-1092.

(25) Klein, C. L.; Scholl, M.; Maelicke, A. Neuronal networks in vitro: Formation and organization on biofunctionalized surfaces. J. Mater. Sci.: Mater. Med. 1999, 10, 721-727.

(26) Buttiglione, M.; Vitiello, F.; Sardella, E.; Petrone, L.; Nardulli, M.; Favia, P.; d'Agostino, R.; Gristina, R. Behaviour of SH-SY5Y neuroblastoma cell line grown in different media and on different chemically modified substrates. Biomaterials 2007, 28 (19), 29322945.

(27) Dwane, S.; Durack, E.; Kiely, P. a. Optimising parameters for the differentiation of SH-SY5Y cells to study cell adhesion and cell migration. BMC Res. Notes 2013, 6, 366.

(28) Krishnamoorthy, M.; Hakobyan, S.; Ramstedt, M.; Gautrot, J. E. Surface-Initiated Polymer Brushes in the Biomedical Field: Applications in Membrane Science, Biosensing, Cell Culture, Regenerative Medicine and Antibacterial Coatings. Chem. Rev. 2014, 114, 10976-11026.

(29) Arima, Y.; Iwata, H. Effect of wettability and surface functional groups on protein adsorption and cell adhesion using well-defined mixed self-assembled monolayers. Biomaterials 2007, 28 (20), 30743082.

(30) Bernards, M. T.; Cheng, G.; Zhang, Z.; Chen, S.; Jiang, S. Nonfouling polymer brushes via surface-initiated, two-component atom transfer radical polymerization. Macromolecules 2008, 41, 42164219.

(31) Ramstedt, M.; Cheng, N.; Azzaroni, O.; Mossialos, D.; Mathieu, H. J.; Huck, W. T. S. Synthesis and characterization of poly(3sulfopropylmethacrylate) brushes for potential antibacterial applications. Langmuir 2007, 23, 3314-3321.

(32) Zhu, B. Surface initiated polymerisation for applications in materials science. Ph.D Thesis, Loughborough University, Loughborough, UK, 2012.

(33) Wei, X.; Ngai, T. Ion-induced hydrophobic collapse of surfaceconfined polyelectrolyte brushes measured by total internal reflection microscopy. Polym. Chem. 2012, 3 (8), 2121.

(34) Edmondson, S.; Osborne, V. L.; Huck, W. T. S. Polymer brushes via surface-initiated polymerizations. Chem. Soc. Rev. 2004, 33, $14-22$.

(35) Shirley, D. A. High-resolution x-ray photoemission spectrum of the valence bands of gold. Phys. Rev. B 1972, 5 (12), 4709-4714.

(36) Quent, V. M. C.; Loessner, D.; Friis, T.; Reichert, J. C.; Hutmacher, D. W. Discrepancies between metabolic activity and DNA content as tool to assess cell proliferation in cancer research. J. Cell. Mol. Med. 2010, 14, 1003-1013.

(37) Rampersad, S. N. Multiple applications of alamar blue as an indicator of metabolic function and cellular health in cell viability bioassays. Sensors 2012, 12, 12347-12360.

(38) Livak, K. J.; Schmittgen, T. D. Analysis of relative gene expression data using real-time quantitative PCR and the 2(-Delta Delta C(T)) Method. Methods 2001, 25 (4), 402-408.

(39) Lee, S. J.; Khang, G.; Lee, Y. M.; Lee, H. B. The effect of surface wettability on induction and growth of neurites from the PC-12 cell on a polymer surface. J. Colloid Interface Sci. 2003, 259 (2), 228-235. 
(40) Jiang, S.; Cao, Z. Ultralow-fouling, functionalizable, and hydrolyzable zwitterionic materials and their derivatives for biological applications. Adv. Mater. 2010, 22, 920-932.

(41) Zhang, Z.; Chao, T.; Chen, S.; Jiang, S. Superlow fouling sulfobetaine and carboxybetaine polymers on glass slides. Langmuir 2006, 22 (24), 10072-10077.

(42) Wan, F.; Pei, X.; Yu, B.; Ye, Q.; Zhou, F.; Xue, Q. Grafting polymer brushes on biomimetic structural surfaces for anti-algae fouling and foul release. ACS Appl. Mater. Interfaces 2012, 4, 45574565.

(43) Zhou, Z.; Yu, P.; Geller, H. M.; Ober, C. K. The role of hydrogels with tethered acetylcholine functionality on the adhesion and viability of hippocampal neurons and glial cells. Biomaterials 2012, 33, 2473-2481.

(44) Tan, K. Y.; Lin, H.; Ramstedt, M.; Watt, F. M.; Huck, W. T. S.; Gautrot, J. E. Decoupling geometrical and chemical cues directing epidermal stem cell fate on polymer brush-based cell micro-patterns. Integr. Biol. 2013, 5, 899-910.

(45) Olivier, A.; Meyer, F.; Raquez, J. M.; Damman, P.; Dubois, P. Surface-initiated controlled polymerization as a convenient method for designing functional polymer brushes: From self-assembled monolayers to patterned surfaces. Prog. Polym. Sci. 2012, 37, 157-181. 